\title{
Negating effects from sodium profile variations for TMT: the MOR truth wavefront sensor of NFIRAOS
}

David R. Andersen, Rodolphe Conan, Brent Ellerbroek, Glen Herriot, Jean-Pierre Véran

David R. Andersen, Rodolphe Conan, Brent Ellerbroek, Glen Herriot, JeanPierre Véran, "Negating effects from sodium profile variations for TMT: the MOR truth wavefront sensor of NFIRAOS," Proc. SPIE 7015, Adaptive Optics Systems, 70155V (15 July 2008); doi: 10.1117/12.789841

Event: SPIE Astronomical Telescopes + Instrumentation, 2008, Marseille, France 


\title{
Negating effects from Sodium profile variations for TMT: The MOR truth wavefront sensor of NFIRAOS
}

\author{
David R. Andersen**a, Rodolphe Conan ${ }^{\mathrm{b}}$, Brent Ellerbroek ${ }^{\mathrm{c}}$, Glen Herriot ${ }^{\mathrm{a}}$ and Jean-Pierre Véran ${ }^{\mathrm{a}}$ \\ ${ }^{a}$ NRC Herzberg Institute of Astrophysics, 5071 W. Saanich Rd, Victoria, BC, Canada V9E 27; \\ ${ }^{\mathrm{b}}$ University of Victoria, PO Box 3055 STN CSC, Victoria, BC Canada V8W 3P6; \\ ${ }^{\mathrm{c}}$ TMT Observatory Corporation, 2632 E. Washington Blvd, Pasadena, CA USA 91107
}

\begin{abstract}
The Moderate Order Radial (MOR) Truth Wavefront Sensor (TWFS) of NFIRAOS, the facility AO system for TMT, is a visible light order 12x12 subaperture Shack-Hartmann WFS. Its role is to sense radial wavefront errors arising from variations in the Sodium layer profile that are not sensed by the on-instrument near infrared tip-tilt focus wavefront sensor at a sampling frequency on the order of one Herz. It works in concert with the High Order Low bandwidth (HOL) TWFS, which will use a 120x120 subaperture Shack-Hartmann WFS that senses slow variations in telescope flexure and the rotation of the pupil. Top-level requirements for NFIRAOS leave little margin for degradation in sky coverage or additional implementation wavefront errors introduced by the operation of the MOR TWFS. In this paper, we explore MOR TWFS design trade studies on the number of subapertures, sampling rate, the width of the MOR TWFS visible bandpass, and the split in light between the MOR and HOL TWFS, and present a design for a system which meets the top level requirements by not degrading the high sky coverage of NFIRAOS $(50 \%$ sky coverage at the Galactic poles) and rejecting the radial modes with a residual wavefront error of $10 \mathrm{~nm}$.
\end{abstract}

Keywords: Adapive Optics, Extremely Large Telescopes

\section{INTRODUCTION}

NFIRAOS, the facility MCAO system for $\mathrm{TMT}^{1-2}$, contains two visible-light (i.e. light bluewards of 1 micron) Truth Wavefront Sensors (TWFS). Both TWFS will share light from a single bright star in the NFIRAOS 2 arcminute FOV (see Atwood et al. for details of the opto-mechanical design ${ }^{3}$ ). Currently, we plan to direct $95 \%$ of the light to the Moderate Order Radial (MOR) TWFS, the goal of which is to sense radial wavefront distortions arising from variations in the structure of the Sodium profile. These modes are induced due to the lag between the laser guidestar matched filter updates ${ }^{4}$. Defocus will be sensed by the on-instrument WFSs, but the third and fifth radial modes (Zernike polynomials $Z_{11}$ and $Z_{22}$ ) will be sensed by the MOR TWFS. The remainder of the light will be used by the $120 \times 120$ subaperture High Order Low-bandwidth (HOL) TWFS to monitor flexures, thermal distortions, and the shape of the rotating TMT pupil on the timescale of minutes.

This paper focuses on the $12 \times 12$ subaperture MOR TWFS. Ideally, the MOR TWFS would only require light from $600 \mathrm{~nm}$ to $800 \mathrm{~nm}$ (corresponding roughly to the Johnson R-band), thereby allowing light redward of this to be passed to the NFIRAOS client science instruments. At these MOR TWFS operation wavelengths, we do not expect significant image-sharpening. Therefore each subaperture of the MOR TWFS will contain 8x8 0.15 arcsec pixels (The pixel size was chosen to Nyquist sample the best expected seeing of $0.3 \mathrm{arcsec}$ ). We have modeled the MOR TWFS performance based upon the use a low readnoise detector in this wavefront sensor such as the E2V CCD201-20. We estimate the required readout rate of the MOR based on simulations of a single Sodium LIDAR dataset ${ }^{5}$ that indicate the induced radial errors must be corrected on a timescale of $100 \mathrm{~s}^{6-8}$. However as many as 20 (40) measurements need to be made in order to reduce these terms to $10 \%(1 \%)$ of their original value if a relatively low gain $(\mathrm{g} \sim 0.1)$ is employed ${ }^{9}$. This

*david.andersen@nrc-cnrc.gc.ca; phone 1250 363-8708; fax 1250 363-0045

Adaptive Optics Systems, edited by Norbert Hubin, Claire E. Max, Peter L. Wizinowich, Proc. of SPIE Vol. 7015, 70155V, (2008)

0277-786X/08/\$18 doi: $10.1117 / 12.789841$

Proc. of SPIE Vol. 7015 70155V-1 
implies that the MOR TWFS should be able to function well enough to meet its requirements with exposure times between $2.5-5 \mathrm{~s}$.

There are two competing requirements governing the design and performance of the MOR TWFS. The first is that the MOR TWFS will not decrease the NFIRAOS sky coverage to less than $50 \%$, even at the Galactic poles. A strong goal is to have little or no impact on the NFIRAOS sky coverage due to the MOR TWFS. The second requirement is that the MOR TWFS shall sense and be able to reject radial modes with a residual wavefront error of $10 \mathrm{~nm}$. This, as we shall see, requires relatively high signal to noise $(\mathrm{S} / \mathrm{N})$ centroid measurements.

In this paper, we show that the MOR TWFS can be read out every $2 \mathrm{~s}-3 \mathrm{~s}$ with minimal loss in sky coverage and wavefront errors less than $10 \mathrm{~nm}$. In the next section, we determine the limiting magnitude at which the MOR TWFS needs to function. Then we discuss our method for partitioning the $10 \mathrm{~nm}$ of wavefront error and arriving at an expression for the MOR TWFS centroiding error. We then use this formula to determine whether to use the WFS in the high gain/no readnoise mode or the normal mode with modest readnoise, and the optimal number of subapertures for the MOR TWFS. Once these trades have been studied, we will assess the probability distribution of wavefront errors as a function of exposure time. We conclude with a summary and a description of future directions for this work.

\section{MOR TWFS LIMITING MAGNITUDE}

To achieve the top-level requirement of NFIRAOS that it provide $50 \%$ sky coverage even at the Galactic poles, the current plan for NFIRAOS and instruments calls for two near infrared (NIR) tip-tilt (T/T) WFSs and one NIR tip-tiltfocus (T/T/F) WFS located within the NFIRAOS client instruments. Simulations have shown that by using these three NIR WFSs, NFIRAOS will meet the sky coverage requirement with some margin ${ }^{10}$. Here, we examine the degree to which the MOR TWFS will impact sky coverage. Because the MOR TWFS uses the visible (R-band) starlight and the on-instrument low order WFSs use NIR starlight, we adopted the Besançon model ${ }^{11}$, which yields a catalog of stars with optical and NIR colors. We modified the NFIRAOS sky coverage tool ${ }^{12}$ to take as input a large (500 in this case) number of realizations of the star fields at the Galactic pole (rather than having the sky coverage tool generate its own star fields based on star counts). Then, as input to the simulation, we adopted fairly typical input parameters (Table 1). On the output of the simulations, we identified all the simulated star fields that met the requirements that all 3 NIR oninstrument WFSs could be deployed on a star brighter than $\mathrm{J}=22$ and that the residual tip-tilt error be less than 2 mas (Figure 1). For the $60 \%$ of the fields that met this requirement, we identified the brightest R-band star in the field for use with the MOR TWFS. The resultant probability distribution of MOR TWFS guide stars is shown in Figure 2.

Table 1: NFIRAOS sky coverage modeling inputs

\begin{tabular}{|l|l|}
\hline Turbulence Profile & Armazones $50 \%$ profile $\left(\mathrm{r}_{0}=16.1 \mathrm{~cm}\right)^{13}$ \\
\hline TMT Windshake model & 20 mas $(70 \% \text { windshake model })^{10}$ \\
\hline FOV & 10 arcsec NFIRAOS field \\
\hline T/T/F WFS Readnoise & 10 electrons \\
\hline Required number of stars & 3 \\
\hline Limiting Magnitude & $\mathrm{J}=22$ \\
\hline Bandpass & $\mathrm{J}$-band: $\lambda_{0}=1.25 \mu \mathrm{m} ; \Delta \lambda=0.3 \mu \mathrm{m}$ \\
\hline Residual tip-tilt & $2 \mathrm{mas}$ \\
\hline Sky Coverage & $60 \%$ \\
\hline
\end{tabular}

To achieve at least $50 \%$ sky coverage with NFIRAOS at the Galactic Poles, we can reduce the sky coverage another $\sim 15 \%$ (unless other factors, such as higher readnoise NIR detectors or details of the T/T/F WFS design further reduce the current margin). This work implies then that the MOR TWFS needs to work with stars at least as faint as $\mathrm{R}=18.3$. To have very little effect on sky coverage, the MOR TWFS would have to use stars as faint as $\mathrm{R}=20$. 


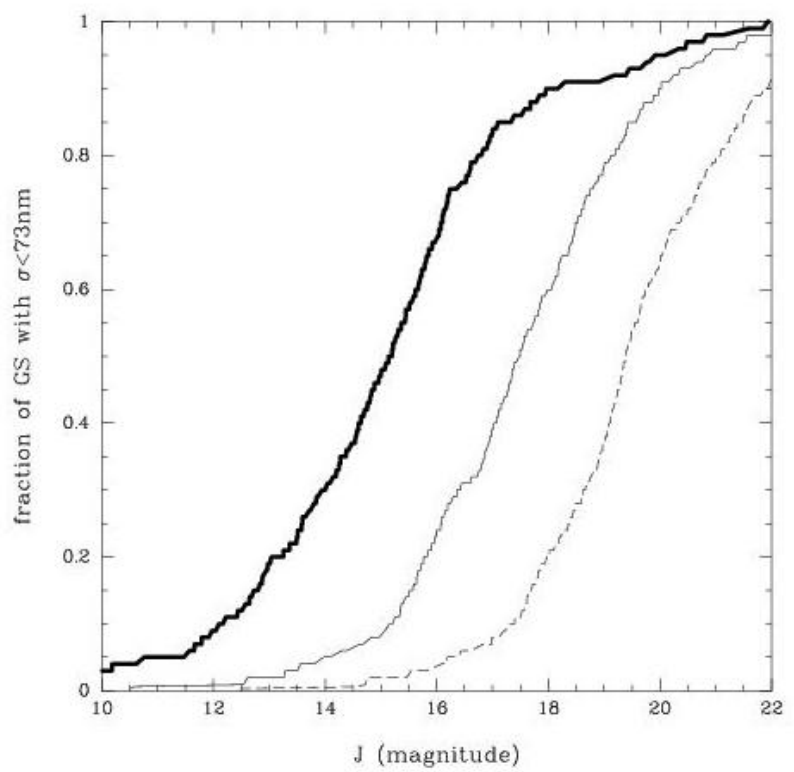

Figure 1: Probability of finding NIR T/T/F star (heavy solid line) and two T/T (thin solid and dashed lines) stars brighter than a given J-band magnitude at the Galactic pole which also meet the NFIRAOS 2mas tip-tilt error budget requirement (satisfied in $60 \%$ of simulated fields; Table 1). The T/T/F and two T/T WFSs are both contained within the NFIRAOS client instruments.

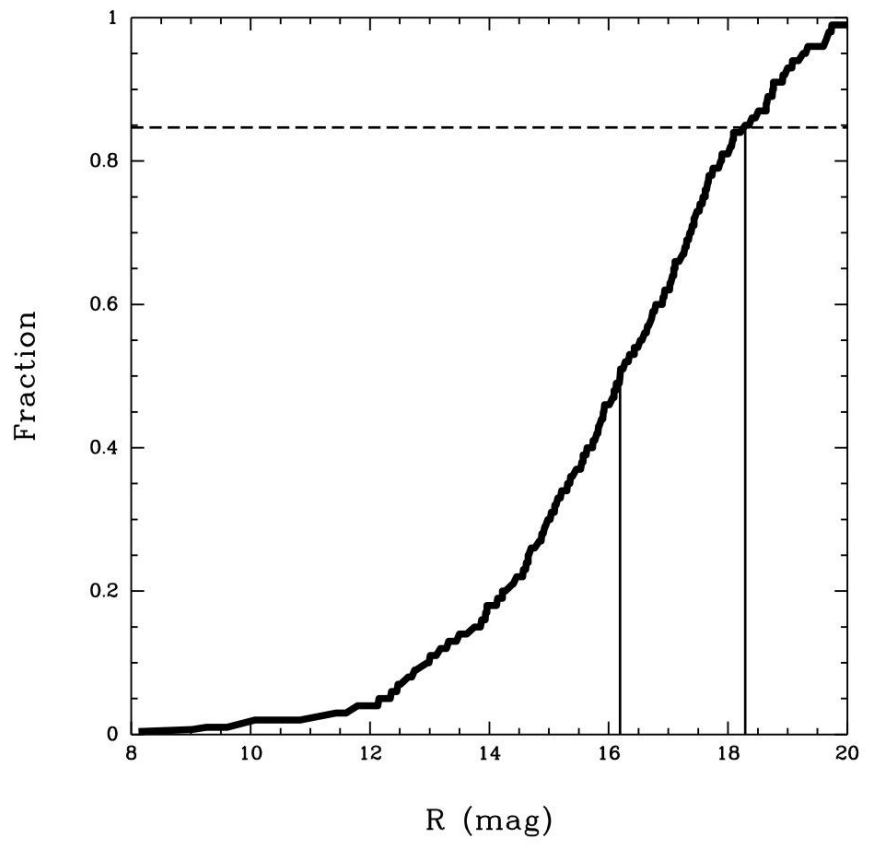

Figure 2: The probability of finding a MOR (and HOL) TWFS star brighter than a given R-band magnitude at the Galactic Pole in cases where the $\mathrm{T} / \mathrm{T} / \mathrm{F}$ probe requirements are met. The median magnitude of a MOR TWFS star will be $\mathrm{R}=16.1$, while the MOR TWFS must be able to operate down to $\mathrm{R}=18.3$ in order for NFIRAOS to have a total system sky coverage above $50 \%$ at the Galactic pole. If the TWFS can operate with any star brighter than $\mathrm{R}=20$, there will not be a significant impact on the overall NFIRAOS sky coverage. 


\section{MOR TWFS NOISE}

Having established that the MOR TWFS should work using stars as faint as $\mathrm{R}=20$, we turn to examine the MOR TWFS wavefront error requirement. The NFIRAOS high order implementation error budget has allocated just 10nm of residual wavefront error from variations in the Sodium profile. This error will arise from the degree of serve rejection of the radial aberrations and MOR TWFS noise. To first order, we expect that each of these terms will contribute equally to the final $10 \mathrm{~nm}$. Therefore, we expect WFS noise will contribute $\sim 7 \mathrm{~nm}$ of wavefront error. We consider this second noise term here.

\subsection{Noise Variance}

To study the WFS noise of the MOR TWFS, we first need to construct the noise covariance matrix. To do this, we first create an interaction matrix for the MOR TWFS based upon Zernike polynomials. In particular, we have looked at the first two radial modes beyond focus (Figure 3). Inverting the interaction matrix, we produce a reconstructor, $C$. We can use this reconstructor to translate between wavefront errors $\left(\sigma_{\mathrm{a}}\right.$, in $\left.\mathrm{nm}\right)$ to centroiding errors $\left(\sigma_{\mathrm{s}}\right.$, in arcseconds), using the equation:

$$
\sigma_{\mathrm{a}}^{2}=<a a^{\mathrm{T}}>=<C s s^{\mathrm{T}} C^{\mathrm{T}}>
$$
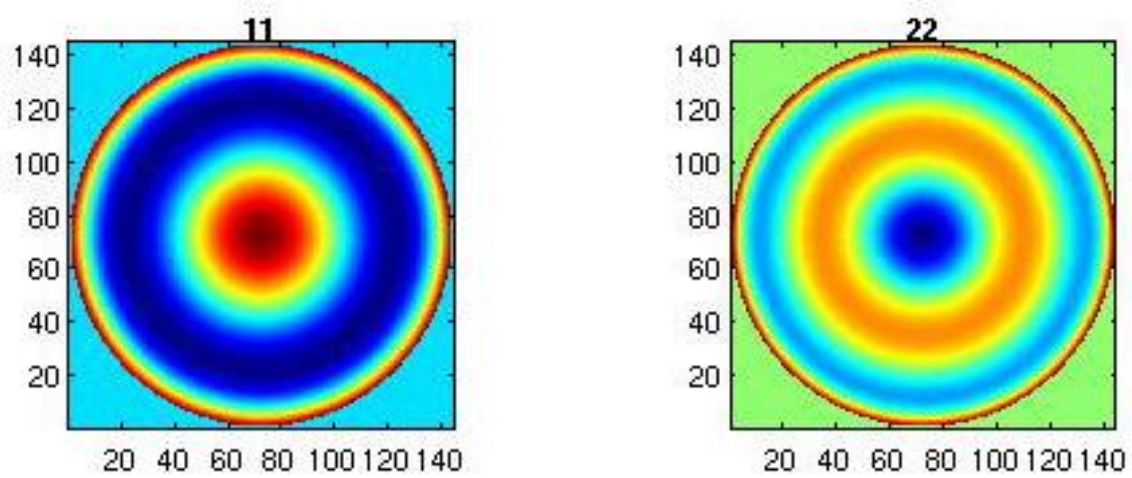

Figure 3: Third and fifth order spherical aberrations are described by the two radial Zernike polynomials past defocus.

$$
Z_{11}=1-6 \rho^{2}+6 \rho^{4} . Z_{22}=-1+12 \rho^{2}-30 \rho^{4}+20 \rho^{6}
$$

The quantity $\left\langle s s^{\mathrm{T}}\right\rangle=\sigma_{\mathrm{s}}{ }_{\mathrm{s}}\langle w\rangle$ where the matrix $w$ is diagonal and very close to the identity matrix, differing only to account for the illumination pattern on different subapertures. For the source-limited case in which we find ourselves, the diagonal elements of $w$ are inversely proportional to the illuminated fraction of the subaperture. Replacing the matrix $w$ with the identity matrix temporarily, we examine the covariance matrix $\left\langle C C^{\mathrm{T}}\right\rangle$. For a sufficiently large number of subapertures ( $12 \times 12$ is adequate here), this covariance matrix for the first 22 Zernike polynomials takes the form shown in Figure 4. The diagonal elements decrease monotonically, in step with theory ${ }^{14}$. As a verification that the tools work properly, a MOR TWFS with just $4 \times 4$ subapertures exhibits a much different trend, as the pupil is insufficiently sampled to sense 22 Zernike modes. A wavefront sensor with $6 \times 6$ subapertures will have slightly larger wavefront errors than expected from a well-sampled theoretical WFS, but if the MOR TWFS has at least $12 \times 12$ subapertures, we find excellent agreement with theory. 


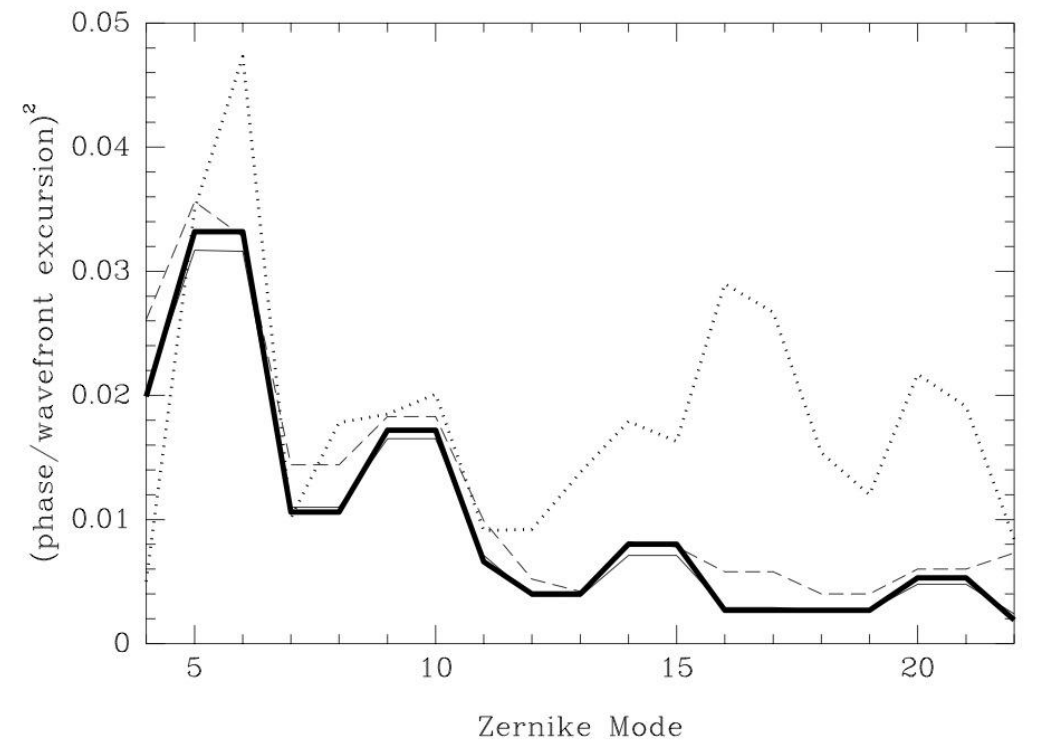

Figure 4: The diagonal elements of the noise variance matrix, $C^{\mathrm{T}}$, for the first 22 Zernike polynomials for WFS with different sampling. The solid black line shows the theoretical value ${ }^{14}$. The dotted line shows the noise variance from a $4 \times 4$ WFS which can not properly measure a fifth order aberration. The variance from a $6 x 6$ WFS is close to theory (dashed line), but excellent agreement is found between a well-sampled theoretical WFS and a 12x12 WFS (solid line).

If we now include the weighting matrix, $w$, and construct $<C w C^{\mathrm{T}}>$ for just the two Zernike modes of interest (Figure 3) using physical units (nm of wavefront error per milliarcsecond centroiding error), we generate the scaling factor between centroiding accuracy and wavefront error as a function of the number of subapertures (Figure 5).

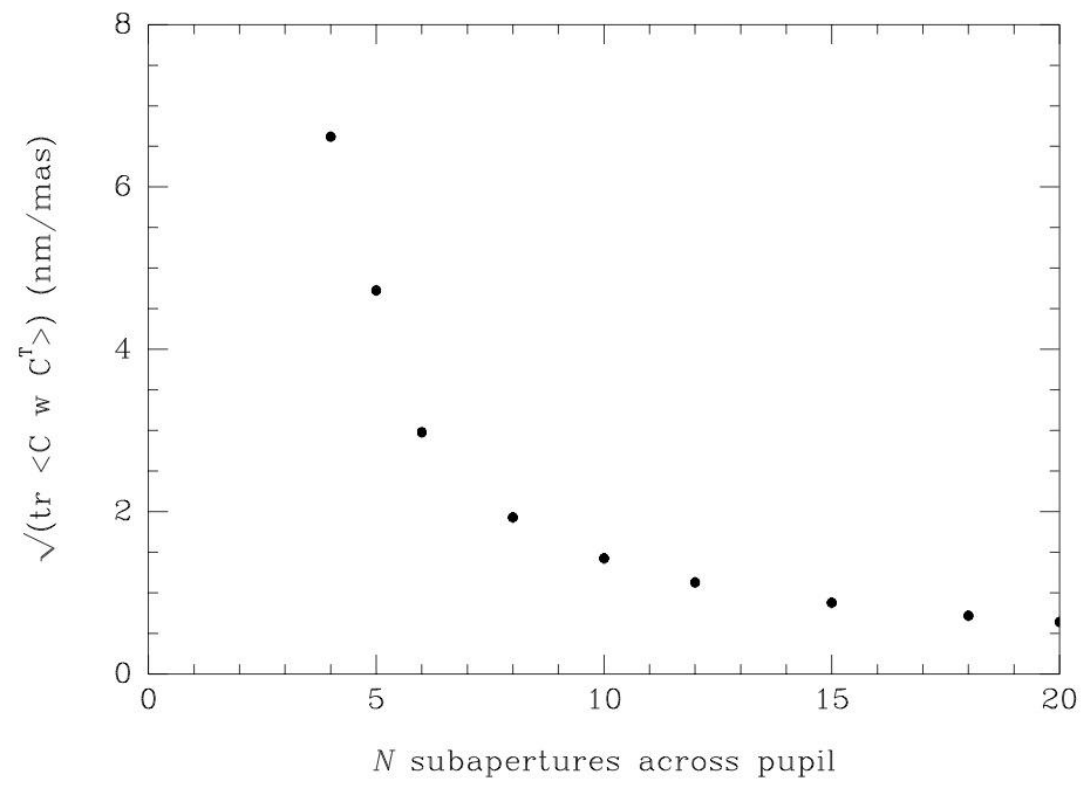

Figure 5: The square root of the trace of the noise variance matrix $<\mathrm{C} \mathrm{w} \mathrm{C}^{\mathrm{T}}>$ for the sum of Zernike modes 11 and 22 decreases as more subapertures are added to the MOR TWFS. While the requirement on centroiding accuracy decreases for an increasing number of subapertures, the light is split more ways as well. We will explore the optimal number of subapertures of the MOR TWFS in section 4.2. Note however, that the centroiding accuracy required to reach a $7 \mathrm{~nm}$ wavefront error is a few milliarcseconds - much smaller than the 150mas TWFS pixel size. 


\subsection{Noise Transfer Function}

Before deriving the centroiding accuracy at a given $\mathrm{S} / \mathrm{N}$ for a point source, the noise transfer function (NTF) needs to be taken into account. The NTF describes how the WFS noise is propogated into the residual signal. The servo analysis is performed using a classical Laplace model, assuming as a controller an integrator with a gain of 0.5 and a delay of $10 \mathrm{~ms}$ (the readout time of the E2V CCD-201-20 detector in normal gain mode with $6 \mathrm{e}^{-}$of readnoise). After performing rough calculations of the required exposure times, we calculated what the $|\mathrm{NTF}|$ would be for exposure times between $10 \mathrm{~ms}$ $\left(\mathrm{F}_{\mathrm{s}}=50 \mathrm{~Hz}\right)$ to $1 \mathrm{~s}(1 \mathrm{~Hz})$, finding values between $0.73>|\mathrm{NTF}|>0.54$. Improvements could be made in the model, but these values for the $|\mathrm{NTF}|$ will not change by large factors. As we shall see in the next section, the required MOR TWFS exposure times are for the most part greater than one second, so a constant value of the $|\mathrm{NTF}|=0.54$ is used for most calculations.

\subsection{WFS Centroiding Precision}

By setting $\sigma_{\mathrm{a}}|\mathrm{NTF}|=7 \mathrm{~nm}$, we can now derive the maximum MOR TWFS sampling frequency (minimum required exposure time) required for a given star magnitude and seeing. Using the two modes shown in Figure 3, we are faced with a tight constraint on the centroiding accuracy; if $|\mathrm{NTF}|=0.54$ (long exposure time), then $7 \mathrm{~nm}$ of wavefront error translates into a centroiding accuracy of $\sigma_{\mathrm{s}}=7 \mathrm{~nm} /|\mathrm{NTF}| / \sqrt{ }<C{ }_{w} C^{\mathrm{T}}>=11$ milliarcsecond for a $12 \times 12$ subaperture WFS, which is $\sim 8 \%$ of a pixel.

To determine the centroiding variance, we assume the point source is seeing limited, with a PSF approximated by a Gaussian that is well-centered on 4 pixels (This is the worst case for determining centroiding accuracy ${ }^{15}$ ). We employ a $10 \%$ threshold in our calculation of the weighted mean centroid. Using these approximations, we can simply propogate the flux errors to arrive at an analytic expression for the centroiding variance:

$$
\sigma_{\mathrm{s}}^{2}=\sum\left[\left(x_{i}-x_{c e n}\right)^{2}\left(f_{i}+\mathrm{B}+\mathrm{D}+\mathrm{RN}^{2}\right)\right] /\left(\sum f_{i}\right)^{2}+\sum\left[f_{i}\left(x_{i}-x_{c e n}\right)\right]^{2} /\left(\sum f_{i}\right)^{4} \sum\left(f_{i}+\mathrm{B}+\mathrm{D}+\mathrm{RN}^{2}\right)
$$

where $\sigma_{\mathrm{s}}$ is the centroiding error in one dimension, $D$ is the dark current per pixel and $\mathrm{RN}$ is the read noise. The number of source photons, $f_{i}$, depends on the magnitude, the seeing and the exposure time, while the number of background photons, $B$, depends only on the exposure time. Characteristics of the bandpass and detector are listed in Table 2 . For a 12x12 MOR TWFS observing a $\mathrm{R}=20$ star, we reach the 11 milliarcsecond centroiding precision corresponding to $7 \mathrm{~nm}$ of wavefront error in $4.3 \mathrm{~s}(\mathrm{~S} / \mathrm{N} \sim 25)$.

\section{MOR TWFS DESIGN TRADE STUDIES}

In this section, we explore how the MOR TWFS exposure time required to reach $7 \mathrm{~nm}$ of noise-induced wavefront error for a $\mathrm{R}=20$ star varies depending on the CCD gain mode and the number of subapertures across the pupil.

\subsection{CCD Gain mode}

We explore whether the E2V CCD201-20 detector (Table 2) would be better used in the high gain, low readnoise mode $(\mathrm{RN}=0.1$ electrons) or in the normal gain, modest readnoise mode ( $\mathrm{RN}=6$ electrons for a reasonable readout time). While it seems obvious that a lower readnoise is better, all the photon noise terms (source, background and dark current terms) will increase by $\sqrt{2}$ if the CCD201 is used in the high gain mode. In fact, we find for a $12 \times 12$ subaperture MOR TWFS that it is advantageous to operate the detector in its normal gain mode and take the readnoise penalty; in the high gain mode, a 5.6s exposure is required (compare to $4.3 \mathrm{~s}$ cited above required in the normal gain mode). To reach the $\mathrm{S} / \mathrm{N}$ needed to get sufficient centroiding accuracy, the MOR TWFS is source-limited in terms of noise. This is true even for the faintest star likely to be used. In bad seeing, where the stellar flux is spread over more pixels, readnoise becomes more important and the exposure time differences between the high and normal gain modes will decrease, but the $\mathrm{S} / \mathrm{N}$ will still dictate that the spots are source-limited and therefore the normal gain mode is still more advantageous. If the number of subapertures in the MOR TWFS were increased, then the high gain mode would be favored (as we shall see in the next subsection). 
Table 2: MOR TWFS bandpass and E2V CCD201-20 detector characteristics

\begin{tabular}{|l|l|}
\hline Sky Background & $\mathrm{R}=20$ mag/sqr arcsecond \\
\hline Bandpass & Johnson R-band: $\lambda_{0}=700 \mathrm{~nm} ; \Delta \lambda=220 \mathrm{~nm}$ \\
\hline Pixel Scale & 0.15 arcsec \\
\hline Throughput & $\begin{array}{l}52 \%[91 \%(\text { atmosphere)x91\%(telescope)x70\% } \\
\text { (NFIRAOS)x95\%(beamsplitter)x95\%(QE)] }\end{array}$ \\
\hline Readnoise & 6 electrons (normal gain mode) \\
\hline Dark Current & 0.04 e/pix/s (detector cooled to -60C) \\
\hline Readout Time & $10^{5}$ pixels in $10 \mathrm{~ms}$ \\
\hline
\end{tabular}

\subsection{Optimal Geometry}

In this section, we determine the minimum required exposure time required to reach $7 \mathrm{~nm}$ of noise induced wavefront error for a $\mathrm{R}=20$ star under median image quality conditions ( 0.6 arcseconds FWHM) for different MOR TWFS geometries. Figure 6 shows that the exposure time is minimal for $12 \times 12$ subapertures across the pupil. The minimum is quite shallow, however, and little difference is found if $8 \times 8$ to $15 \times 15$ subapertures were used. The optimal solution could change slightly based on the exact readnoise of the detector and the seeing (i.e., across how many pixels is the source flux spread); if better image quality were expected, the optimal number of subapertures across the pupil would increase. Figure 6 also clearly demonstrates that the high gain mode is favored if more than 20x20 subapertures are used for the MOR TWFS. However, the minimum required exposure time for a 40x40 MOR TWFS operating in the high gain mode is $10 \%$ larger than the minimum required exposure time for the baseline $12 \times 12$ MOR TWFS. Increasing the number of subapertures beyond 40x40 will start to further increase the minimum required exposure time.

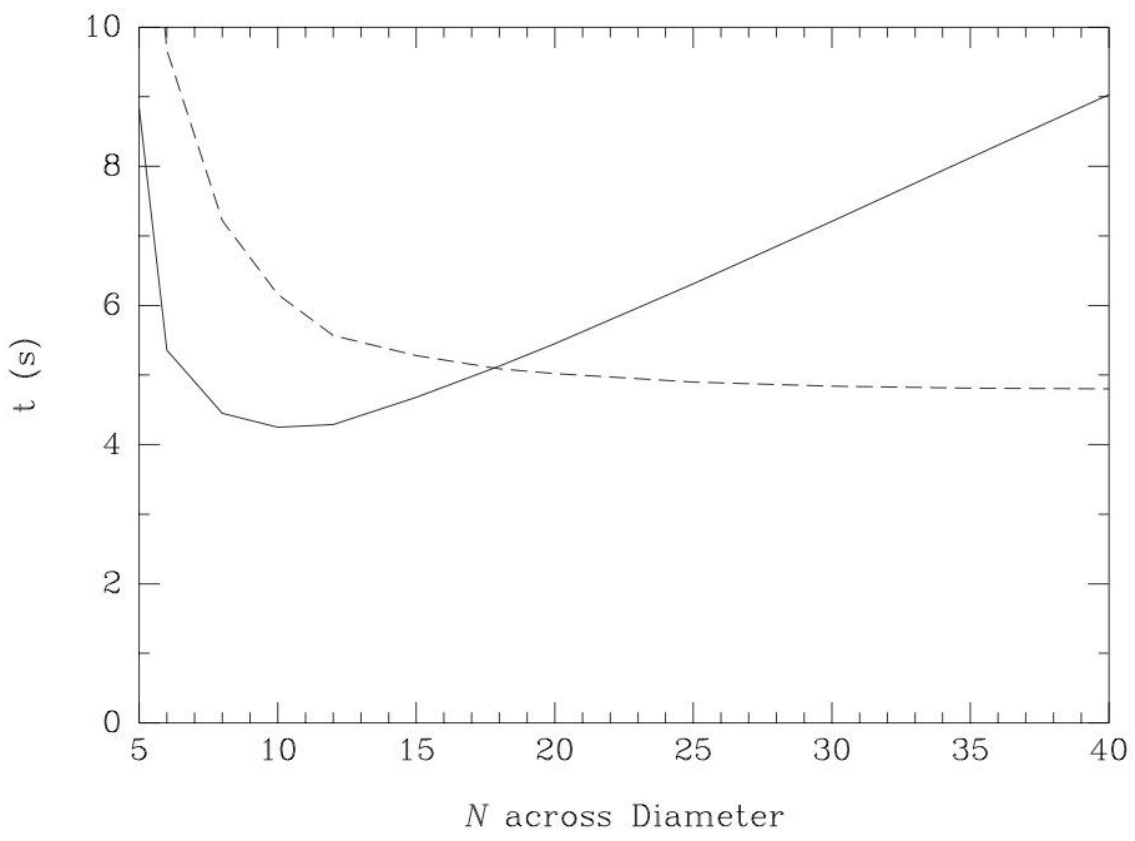

Figure 6: Minimum exposure required to reach a centroiding accuracy corresponding to $7 \mathrm{~nm}$ of noise-induced wavefront error versus the number of subapertures across the MOR TWFS using the CCD201 in high gain mode (low readnoise mode; dashed line) and normal gain mode ( $6 \mathrm{e}^{-}$readnoise mode; solid line). Calculations assume $\mathrm{R}=20$ magnitude sources and median image quality $(0.6$ arcseconds). The minimum is found for a WFS with $12 \times 12$ subapertures, but the minimum is broad. Any number between 8 and 15 gives a comparable result. The optimal solution will depend on the details of the image quality and exact readnoise of the detector. If the image quality is poor, we find that the optimal number of subapertures will decrease slightly, as the flux is spread over more pixels and readnoise will be more important. 


\section{MOR TWFS PERFORMANCE}

Having established the optimal geometry and gain mode for the MOR TWFS, we can now combine our sky coverage analysis of section 2 with the WFS noise analysis of section 3 to study the performance trade-offs between sky coverage, wavefront error and minimum MOR TWFS exposure time. We use the probability distribution in Figure 2 to determine the probability of achieving a given noise-induced wavefront error from the MOR TWFS for different exposure times. We show the results in Figure 7 for median image quality $\left(0.6\right.$ arcsecond seeing corresponding to $\left.r_{0}=16.1 \mathrm{~cm}\right)$ using a 12x12 MOR TWFS with the detector operating in normal gain mode with 6 electrons of readnoise.

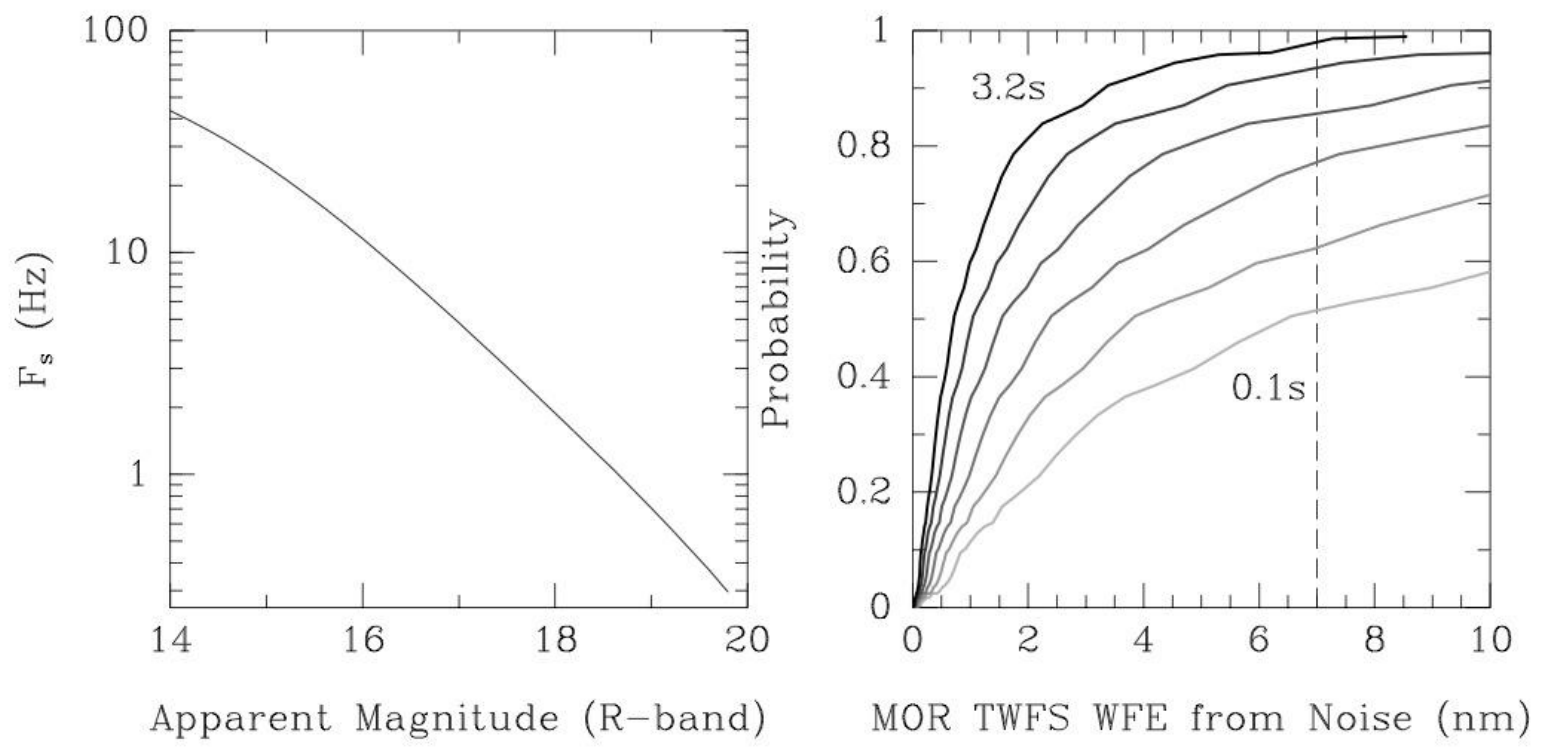

Figure 7: Left Panel: Sampling frequency corresponding to 7nm of WFS noise error versus apparent magnitude of the $12 \times 12$ MOR TWFS star assuming median image quality (0.6 arcsec FWHM) and a $10 \mathrm{~ms}$ readout time. Right Panel: Probability of achieving a given noise-induced wavefront error from the MOR TWFS for different exposure times with median image quality. We assume only R-band light is used by the MOR TWFS (Table 2). Each line represents a doubling of the exposure time from $0.1 \mathrm{~s}$ to $3.2 \mathrm{~s}$. The goal is to achieve less than $7 \mathrm{~nm}$ of wavefront error due to noise (vertical dashed line).

The results are promising; well over $95 \%$ of the time the minimum exposure time is less than $3 \mathrm{~s}$ that should be sufficiently short enough to sample and correct the radial wavefront errors induced by the changing Sodium profile, even for a low gain (section 1). More than half the time, the minimum required exposure will be much shorter than one second.

Up until this point, we have only considered a MOR TWFS accepting R-band light (600-800nm). While this limited bandpass is sufficient to meet both the sky coverage and wavefront error budgets of NFIRAOS, remember that the MOR TWFS shares the light of one star with the HOL TWFS. The HOL TWFS will be photon starved because it has $120 \times 120$ subapertures and in the baseline design only receives $\sim 2.5 \%$ of the light emitted from the star (a beamsplitter sitting directly in front of the MOR and HOL TWFSs directs $95 \%$ of the light to the MOR and directs only $5 \%$ of the remaining light to the HOL TWFS). To increase the light available to the HOL TWFS, we consider the possibility of replacing the beamsplitter with a dichroic. In this scenario all the light redwards of $800 \mathrm{~nm}$ would be directed to the HOL TWFS and only light with wavelengths between $800 \mathrm{~nm}$ and $950 \mathrm{~nm}$ would be passed to the MOR TWFS. At these longer wavelengths, the MOR TWFS can benefit from two effects: potential MOR TWFS targets are predominantly red (Figures 8-9) so more source photons would enter the MOR TWFS, but more importantly, the MOR TWFS could be designed to take advantage of image sharpening from the adaptive optics system. Making this design choice would negatively impact the NFIRAOS client instrument science cases in that only light redwards of 1 micron would be available to the science instruments; these science instruments will be blind to important spectral features such as the Calcium triplet $(850 \mathrm{~nm})$ and $\mathrm{H} \alpha$ at redshifts less than 0.5 . 


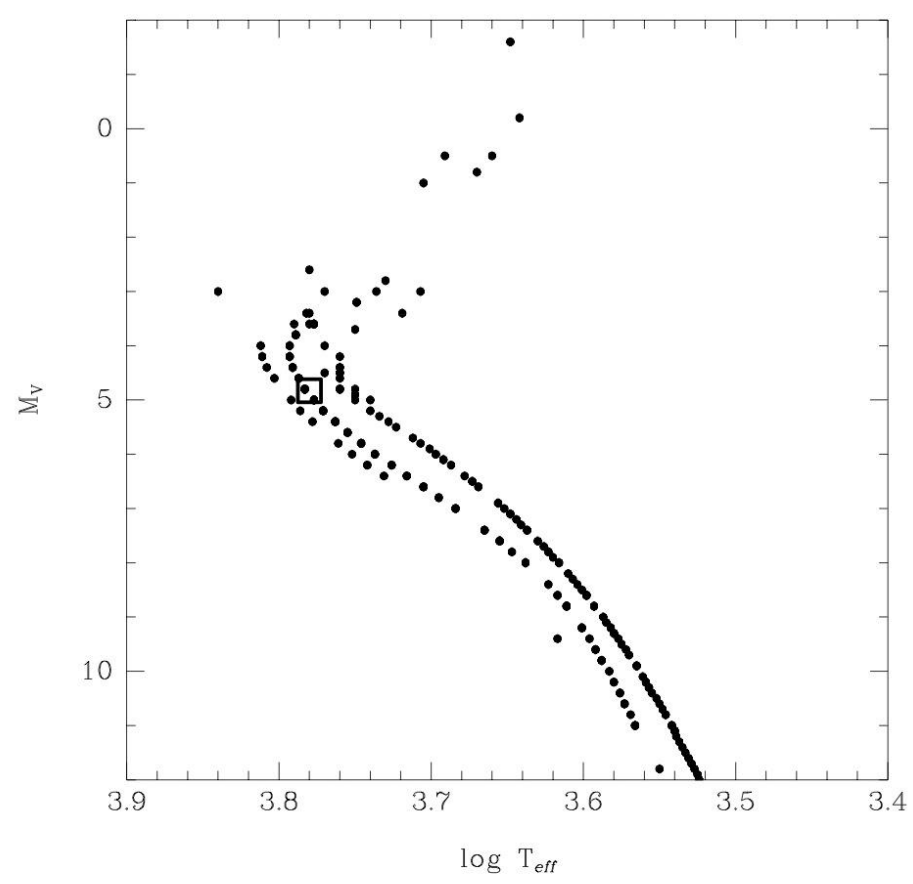

Figure 8: The Hertzsprung-Russell diagram for candidate MOR TWFS targets at the NGP taken from the Besançon simulations. This plot of effective temperature versus absolute magnitude shows that the large majority of these sources are main sequence stars intrinsically fainter and cooler (redder) than our sun (marked with a large square).

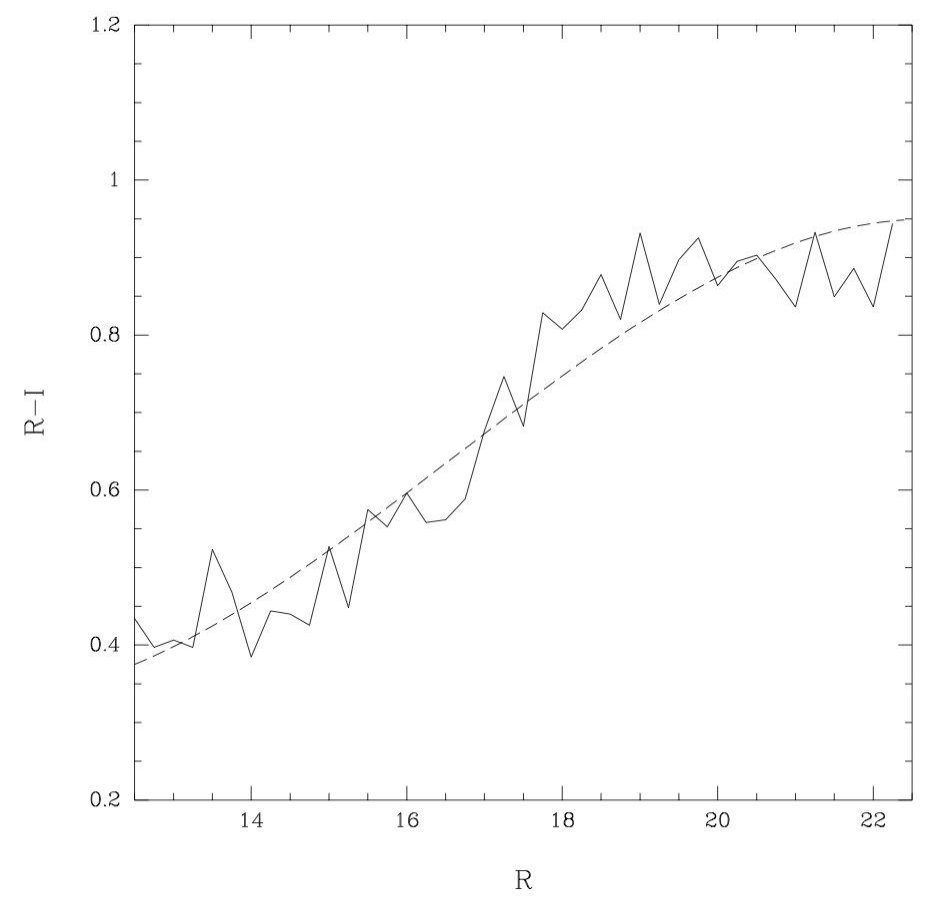

Figure 9: Mean R-I color of potential MOR TWFS targets at the Galactic pole as a function of R-magnitude. We use the $5^{\text {th }}$ order polynomial fit (dashed line) to convert our R-band magnitudes to I-band magnitudes. 
Although this scientific drawback must be kept in mind, the overall performance of NFIRAOS may improve if the HOL TWFS receives more light. This change has little impact on the MOR TWFS performance (Figure 10); even if $1 \%$ of the light from the star is concentrated in the diffraction-limited peak corresponding to a $\mathrm{d}_{0}=2.5 \mathrm{~m}$ subaperture, we find that the minimum exposure time required by the MOR TWFS is comparable to the R-band case (Figure 7). In some cases, however, the images should be sharpened even more resulting in shorter minimum exposure times for the MOR TWFS. If more light is required by the HOL TWFS, we will explore this scenario in greater depth.

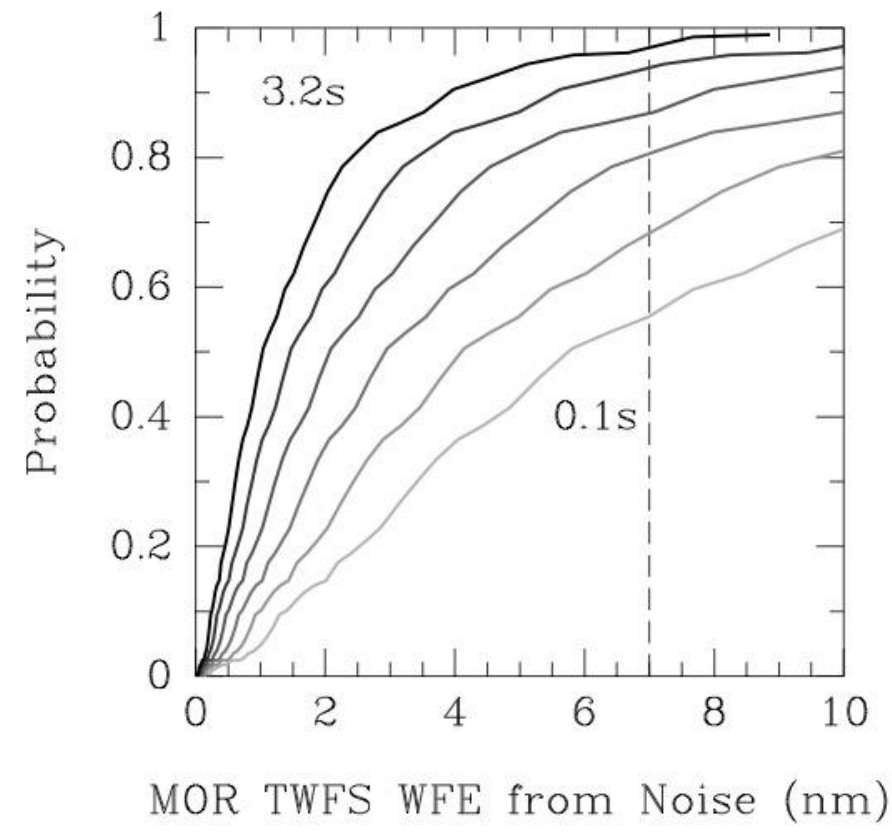

Figure 10: Probability of achieving a given noise-induced wavefront error from a MOR TWFS employing image sharpening for different exposure times and just a $1 \%$ Strehl ratio. Each line represents a doubling of the exposure time from $0.1 \mathrm{~s}$ to $3.2 \mathrm{~s}$. The goal is to achieve less than $7 \mathrm{~nm}$ of wavefront error due to noise (vertical dashed line). Even if just $1 \%$ of the light is in the diffraction-limited core (for $\mathrm{d}_{0}=2.5 \mathrm{~m}$ ) in an I-band MOR TWFS designed to work for diffractionlimited images, we get the same performance as with the R-band seeing-limited MOR TWFS (Figure 7). For slightly higher Strehl ratios, we could achieve markedly shorter minimum exposure times. The great advantage of this approach, however, is that all the light bluewards of 800nm could be sent to the HOL TWFS. Unfortunately, this design choice would negatively impact the science cases of NFIRAOS client instruments. Future work on the role and requirements of the HOL TWFS will have an impact on the MOR TWFS design.

\section{CONCLUSIONS AND FUTURE WORK}

We have evaluated the noise performance of the NFIRAOS MOR TWFS. We have found that it will be much most efficient with $\sim 12 \times 12$ subapertures. The MOR TWFS detector will also work best with a normal gain mode (modest readnoise) setting; the shot noise penalty incurred in the high gain mode is not offset by the low readnoise.

The allocated wavefront error for the NFIRAOS TWFS is small (just 10nm) leading to a very tight requirement on the MOR TWFS spot centroiding accuracy (11mas, just $8 \%$ of a pixel for a $12 \times 12$ WFS). This in turns means that the individual WFS spots have to be detected at relatively high signal to noise $(\mathrm{S} / \mathrm{N} \sim 25)$ for stars as faint as $\mathrm{R}=20$ (to avoid a loss in NFIRAOS sky coverage). The minimum exposure time required by the MOR TWFS to reach this $\mathrm{S} / \mathrm{N}$ is $\sim 3 \mathrm{~s}$ for a $\mathrm{R}=20$ star and median image quality $(0.6$ arcseconds FWHM). Given the results that the radial modes must be corrected on a timescale of 100s based on one Sodium LIDAR dataset, the MOR TWFS will be able to sense and control the radial modes induced by variations in the Sodium layer even if the correction is applied with a low gain (long settling time).

In the next stage of this work, we are working on balancing this important WFS noise error term with other dynamic terms. We are in the process of acquiring and analyzing more Sodium LIDAR profiles to determine matched filter 
update errors over a wider variety of conditions (and with data taken at higher temporal frequency ${ }^{17}$ ). Finally, we are also beginning to explore the magnitude of non-common path aberrations between the LGS WFSs and the MOR TWFS due to variations in relative intensities as a function of height for the Sodium layer and due to the zoom optics settings. These analyses will allow us to refine our design to create a MOR TWFS which optimally senses and negates residual wavefront errors due to changes in the Sodium layer.

\section{REFERENCES}

[1] Ellerbroek, B. et al. "Progress towards developing the TMT adaptive optical systems and their components," Proc. SPIE 7015 (2008)

[2] Herriot, G. et al. "NFIRAOS - TMT's initial adaptive optics system", Proc. SPIE 7015 (2008)

[3] Atwood, J., Byrnes, P. and Herriot, G. "Present Optical and Mechanical Design Status of NFIRAOS for TMT," Proc. SPIE 7015 (2008)

[4] Gilles, L., \& Ellerbroek, B.L., "Constrained Matched Filtering for Extended Dynamic Range and Improved Noise Rejection for Shack-Hartmann Wavefront Sensing," Opt. Lett. 33 (2008)

[5] Saul-Davis, D., Hickson, P. Herriot, G., She, C.-Y., "Temporal variability of the telluric sodium layer," Opt. Lett. $31,3369(2006)$

[6] Gilles, L. Wang, L. \& Ellerbroek, B., "Wavefront Error Budget Development for the Thirty Meter Telescope Laser Guide Star Adaptive Optics System," Proc. SPIE 7015 (2008)

[7] Conan, R., Herriot, G., Lardière, O., Jackson, K., Bradley, C., "Modeling of the Thirty Meter Telescope matched fileter-based wavefront sensing," Proc. SPIE 7015 (2008)

[8] Wang, L., Ellerbroek, B., \& Gilles, L., "Impact of sodium layer profile variability upon adaptive optics systems performance," submitted to Opt. Exp. (2008)

[9] Poyneer, L., "Integrated simulations of Calibration and AO," Appendix 1 of the Gemini Planet Imager (GPI) critical design report (2008)

[10] Wang, L., Ellerbroek, B., Véran, J.-P., Sinquin, J.-C., "Evaluating Sky Coverage for the NFIRAOS Tip/Tilt Control Architecture," Proc. SPIE 7015 (2008)

[11] Clare, R.M., Ellerbroek, B.L., Herriot, G. Véran, J.-P., “Adaptive optics sky coverage modeling for extremely large telescopes," Appl. Opt. 45, 8964 (2006)

[12] Schöck, M. et al., "Status of the thirty meter telescope site selection program," Proc. SPIE 7012 (2008)

[13] Robin, A.C., Reylé, C., Derriére, S., Picaud, S., "A synthetic view on structure and evolution of the Milky Way," A\&A, 409, 523 (2003)

[14] Rigaut, F. \& Gendron, E., "Laser guide star in adaptive optics - the tilt determination problem," A\&A, 261, 677 (1992)

[15] King, I.R., "Accuracy of measurement of star images on a pixel array," PASP, 95, 163 (1983)

[16] Gemini Mauna Kea background model, http://www.gemini.edu/sciops/ObsProcess/obsConstraints/ocSkyBackground.html

[17] Pfrommer, T., Hickson, P., She, C.-Y., Vance, J., "Lidar experiment for high spatio-temporal resolution of the mesospheric sodium layer," Proc. SPIE 7018 (2008) 